Case Report

\title{
Autoimmune hemolytic anemia- an initial presentation in childhood systemic lupus erythematosus
}

\author{
Giriyan S.S. ${ }^{1}$ Priya $^{2}$, Reddy P. ${ }^{3}$ \\ ${ }^{1}$ Dr Sujata S. Giriyan, Professor and Head, ${ }^{2}$ Dr. Priya, Post Graduate, ${ }^{3}$ Dr. Reddy P.; all authors are affiliated with \\ Department of Pathology, Karnataka Institute of Medical Sciences, Hubballi, Karnataka, India.
}

Corresponding Author: Dr Priya, Department of Pathology, Karnataka Institute of Medical Sciences, Hubballi, Karnataka, India. E-mail: mayurirao0116@gmail.com

\begin{abstract}
Systemic Lupus Erythematosus (SLE) is a chronic autoimmune disease that can involve any organ system. It predominantly affects women aged 20 to 40 years. In $10 \%$ to $15 \%$ of patients, the diagnosis is first established during childhood. Childhood SLE is greater in severity, abrupt with atypical presentation at onset. A 12-year-old girl presented with fever of 8 days and vomiting of 2 days duration. On examination there was pallor and hepatospleenomegaly. Hematologic investigations showed picture of hemolytic anemia. RBCs showed auto-agglutination. Suspecting autoimmune hemolytic anemia, Direct Coombs test was done and was positive. Patient later developed malar rash and hematuria. Suspecting the cause of autoimmune hemolytic anemia to be SLE, Antinuclear antibody, Anti double stranded DNA antibody, Anti Smith antibody were done and showed positive result. Hence the diagnosis was confirmed to be Autoimmune Hemolytic Anemia Due to Systemic Lupus Erythematosus.
\end{abstract}

Keywords: Anti Double Stranded DNA antibody, Antinuclear antibody, Anti Smith antibody, Auto agglutination, Hemolytic anemia.

\section{Introduction}

A 12 year old girl presented with fever and vomiting. On examination there was pallor and hepatospleenomegaly. Peripheral smear showed features of hemolytic anemia and RBCs auto agglutination. Suspecting immune mediated cause for auto agglutination of RBCs direct Coombs test was done which showed positive result $[1,2]$. Diagnosis of autoimmune hemolytic anemia was made. In course patient developed malar rash and hematuria hence, anti nuclear antibody, anti double stranded DNA antibody, anti Smith antibody were done which showed positive results and a final diagnosis of autoimmune hemolytic anemia due to systemic lupus erythematosus was done.

\section{Case Report}

A 12-year-old girl presented with complaints of fever in the last 8 days and vomiting since 2 days. On general physical examination pallor and hepatospleenomegaly were noted. Complete hemogram showed anemia and thrombocytopenia. Peripheral smear showed macrocytic anemia with thrombocytopenia. To rule out the cause of infection causing anemia and thrombocytopenia investigations like HIV, $\mathrm{Hb} \mathrm{s} \mathrm{Ag} \mathrm{(Hepatitis} \mathrm{B} \mathrm{surface} \mathrm{antigen),} \mathrm{Dengue} \mathrm{NS-1} \mathrm{antigen,} \mathrm{Malaria} \mathrm{tests}$ (rapid and peripheral smear) and blood culture for Brucella were done and they were all negative.

Renal functional tests and liver functional tests were normal. Serial complete hemograms of the patient showed decreasing hemoglobin level and platelet count. Patient was given blood transfusion. After blood transfusion, the peripheral smear showed Dimorphic anemia with Thrombocytopenia (RBC's were macrocytic and microcytic hypochromic with moderate anisopoikilocytosis showing ovalocytes, microspherocytes, occasional nucleated RBC's 13 per 100 WBC's) as shown in figure 1. Due to the presence of microspherocytes, hemolytic anemia was suspected and osmotic fragility test was done which was seen to be increased.

Manuscript received: $23^{\text {th }}$ August 2019

Reviewed: $3^{\text {rd }}$ September 2019

Author Corrected: $8^{\text {th }}$ September 2019

Accepted for Publication: $13^{\text {rd }}$ September 2019 


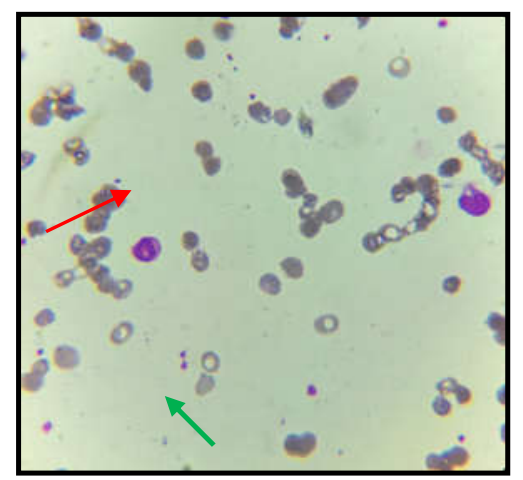

\section{Case Report}

Figure-1: Peripheral smear showing microspherocytes (red arrow) and microcytic hypochromic RBCs (green arrow). (100X, Leishman stain).

Patient received another blood transfusion and post transfusion there was slight increase in hemoglobin level and the peripheral smear showed the same picture as the above but with increase in polychromatophils and nucleated RBC's and some areas also showed agglutination of RBC's as shown in figure 2. Reticulocyte count was seen to be increased to $15 \%$, shown in figure 3 .

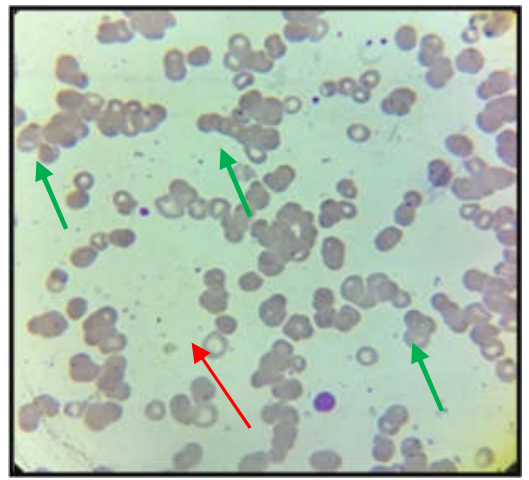

Figure-2: Peripheral smear showing dimorphic picture and microspherocytes(red arrow), agglutination of RBC's (green arrow). (100X, Leishman stain).

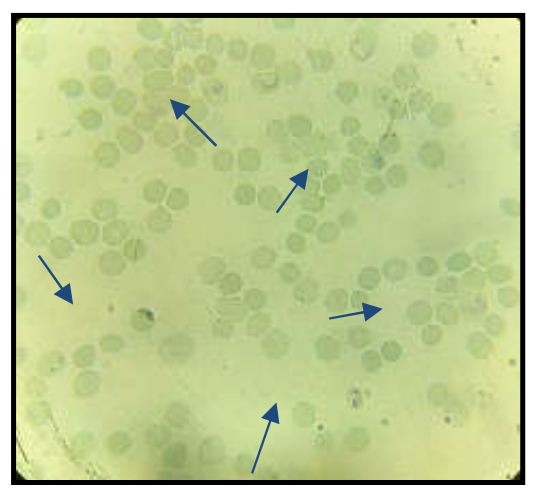

Figure-3: Peripheral smear showing increase number of Reticulocytes (blue arrow). (100X, Methylene Blue stain).

As the RBCs showed agglutination on peripheral smear further evaluation was done. A drop of patient's blood and control blood were taken on separate slides, placed a cover slip above and was visualized under microscope. Both the patient's and the control blood showed agglutination of RBCs, as shown in figure 4 and 6 . To differentiate between rouleux formation and auto agglutination of RBCs, a drop of patient's blood was mixed with a drop of normal saline, cover slip was placed and visualized under microscope, similar procedure was done for control blood. Patient's blood showed persistence of agglutinated RBCs even after normal saline wash, shown in figure 5, where as in the control blood the agglutination disappeared after the normal saline wash, shown in figure 7. This confirms that the patient's RBCs are showing auto agglutination. 


\section{Case Report}

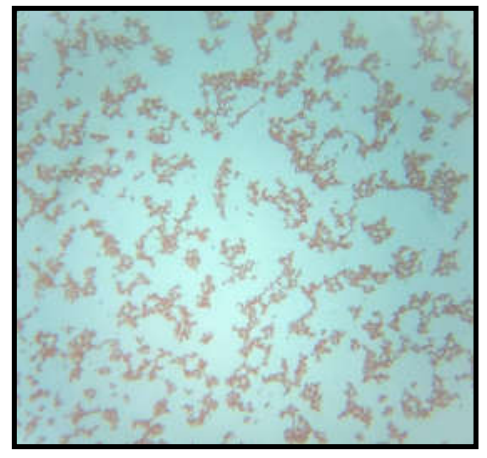

Figure-4: Patient's RBCs showing agglutination.

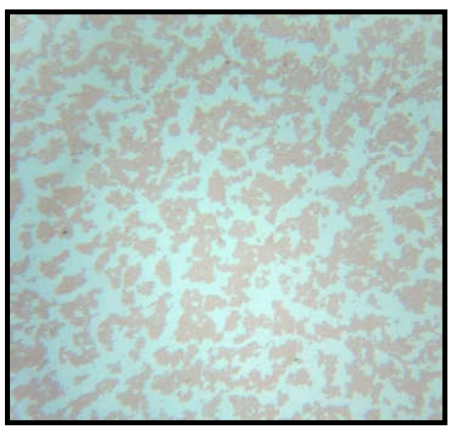

Figure-5: Patient's RBCs showing agglutination after normal saline wash.

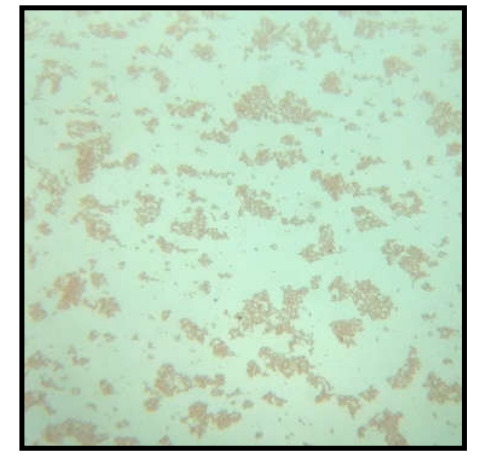

Figure-6: Control blood RBCs showing agglutination.

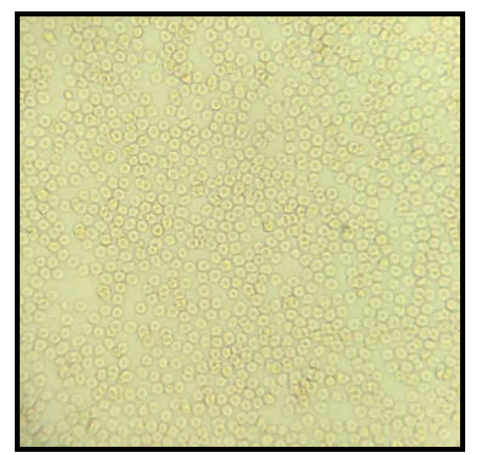

Figure-7: Disappearance of agglutination of RBCs in control blood after normal saline wash.

To confirm for auto agglutination of RBCs and to find the cause of auto agglutination, Direct Coombs test was done which showed positive result. This confirmed the immune mediated cause for auto agglutination of RBCs. Bone marrow studies showed Megaloblastic Erythroid Hyperplasia. Serum Ferritin, Serum Lactate Dehydrogenase were raised. This ruled out aplastic anemia and iron deficiency anemia. 


\section{Case Report}

From all the above performed investigations the diagnosis was narrowed down to autoimmune hemolytic anemia. Patient during the hospital stay developed malar rash and hematuria.

Suspecting the cause of the autoimmune hemolytic anemia to be SLE, Anti nuclear antibody, Anti double stranded DNA antibody, Anti Smith antibody were done which showed positive results. Hence final diagnosis of autoimmune hemolytic anemia due to systemic lupus erythematosus was made.

\section{Discussion}

Systemic Lupus Erythematosus (SLE) is a chronic autoimmune disease that can involve any organ system and may lead to significant morbidity and even mortality [1].

It is characterized by a wide variety of clinical features and presence of numerous auto-antibodies, circulating immune complexes and widespread immunologically determined tissue damage [2]. Hematologic manifestations are often the presenting features seen in SLE, and they are anemia of chronic disease (70\%), leucopenia $\quad(65 \%), \quad$ lymphopenia $\quad(50 \%)$, thrombocytopenia (15\%), lymphadenopathy (15\%), spleenomegaly (15\%), hemolytic anemia (10\%) [3].

According to a study on childhood-onset SLE done by Meunier BB et al [4], they found that malar rash, arthritis and fever were the most common manifestations at presentation. In the present case fever and hematological manifestations (hemolytic anemia, thrombocytopenia) were the initial manifestations at presentation.

Anemia in SLE is caused by immune mediated process (antibodies and/or complement) that bind to red cells, leading to their premature destruction is called immune hemolytic anemia. Autoimmune hemolytic anemia is characterized by production of antibodies against own erythrocyte antigens. Antibodies produced may be warm reacting or cold reacting [5].

In the present case anemia was the initial presenting feature of the disease. The diagnosis of hemolytic anemia was made based on peripheral smear picture, osmotic fragility test, reticulocyte count, bone marrow examination, hepatospleenomegaly.

Autoimmunity was found to be the cause for hemolysis of RBC by Direct Coombs test and detection of auto agglutination of RBCs on peripheral smear. Overt autoimmune hemolytic anemia (AIHA) due to the presence of both immunoglobulin and complement on the red cell is usually associated with some degree of hemolysis characterized by an elevated reticulocyte count, low haptoglobin levels, increased indirect bilirubin concentration and a positive Direct Coombs' test, has been noted in up to $10 \%$ of patients with SLE [5].
In the American College of Rheumatology (ACR) criteria for diagnosis of SLE, hematological changes, though very commonly seen, are not properly evaluated or estimated and are not given enough representation [6].

Hematological investigations also showed thrombocytopenia in the present case. Mild thrombocytopenia (11.5 lakh cells/ dl) has been noted in 25 to $50 \%$ of patients with SLE [5].

Immune mediated platelet destruction is most often the cause for thrombocytopenia in SLE. Immunoglobulin binds to platelets and is phagocytosed in the spleen. Membrane glycoprotein (GP) are most often the target of such antibodies (e.g., GP IIb/ IIIa) [5].

Presence of malar or butterfly rash is the hallmark of SLE and is seen in $60-85 \%$ of children with SLE [1].In the present case, although malar rash was not present initially, it developed during the hospital stay which later lead to the suspicion of SLE. Similarly, the patient also developed hematuria later in the course of the disease. Renal involvement occurs in 50 to $75 \%$ of all childhood SLE patients and more than $90 \%$ of those who will develop renal disease will do so within the first 2 years after diagnosis [1].

The diagnosis of SLE is based on characteristic clinical manifestations and auto antibodies. Antinuclear antibodies (ANA) are positive in $>98 \%$ of patients during the course of disease. High titre Immunoglobulin $\mathrm{G}$ antibodies to double stranded DNA and antibodies to Smith antigen are both specific for SLE and therefore favour the diagnosis in the presence of compatible clinical manifestations.

In the present case, hematological manifestations were the initial features, whereas malar rash, arthritis and fever were the most common manifestations at presentation in a study done by Meunier BB et al [4] on childhood-onset SLE. Hematological work up in this case showed presence of autoimmune hemolytic anemia which is noted in only $10 \%$ of patients with SLE [5]. Malar rash was a late presentation in this case where in $60-85 \%$ children with SLE it is the initial manifestation [1]. 


\section{Conclusion}

Childhood onset SLE is seen in $10-15 \%$ of population. Among the manifestations of SLE, hematologic presentation is most common and autoimmune hemolytic anemia is seen only in $10 \%$ cases of SLE. In the present case presence of $\mathrm{RBC}$ agglutination on the peripheral smear lead to the further evaluation of the case and along with other relevant investigations performed diagnosis of autoimmune hemolytic anemia due to SLE was made.

This case helps to know the variation in the presentation of childhood SLE and in particular the hematological manifestations of the same which has not been properly evaluated in the ACR criteria for the diagnosis of SLE.

\section{References}

1. Levy DM, Kamphuis S. Systemic lupus erythematosus in children and adolescents. Pediatr Clin North Am. 2012;59(2):345-364. doi: 10.1016/j.pcl. 2012.03.007.

2. Janoudi N, Bardisi ES. Haemotologic Manifestations in Systemic Lupus Erythematosus. In: Almoallim H, editor. Systemic Lupus Erythematosis. Intech. Mar 2012.

3. Hahn BH. Systemic Lupus Erythematosus. In: Longo DL, Kasper DL, Jameson JL, Fauci AS, Hauser SL, Loscalzo J, editors. Harrison's Principles of Internal Medicine. $18^{\text {th }}$ edition. United States of America: The McGraw-Hill Companies. 2012; 1:2724- 2735.

4. Meunier B, Armengaud JB, Haddad E, Salomon R, Deschenes $G$, Leblanc $T$ et al. Initial Presentation of Childhood- onset SLE: A French Multicenter Study. J Pediatr. 2005;146(5):648- 653.

5. Smith LA. Haemolytic Anemia: Immune Anemias. In: McKenzie SB, Williams JL, editors. Clinical Laboratory Haematology. $3^{\text {rd }}$ edition. New Jersey: Pearson. 2015; 11:349- 371.

6. Sasidharan PK, Bindya M, Sajeeth Kumar KG. Hematological manifestations of SLE at initial presentation: is it underestimated? ISRN hematology. 2012. 2012: 961872 . doi: 10.5402/2012/961872

\section{How to cite this article?}

Giriyan S.S., Priya, Reddy P. Autoimmune hemolytic anemia- an initial presentation in childhood systemic lupus erythematosus. Trop J Path Micro 2019;5(9):745-749.doi:10.17511/jopm.2019.i09.21. 